
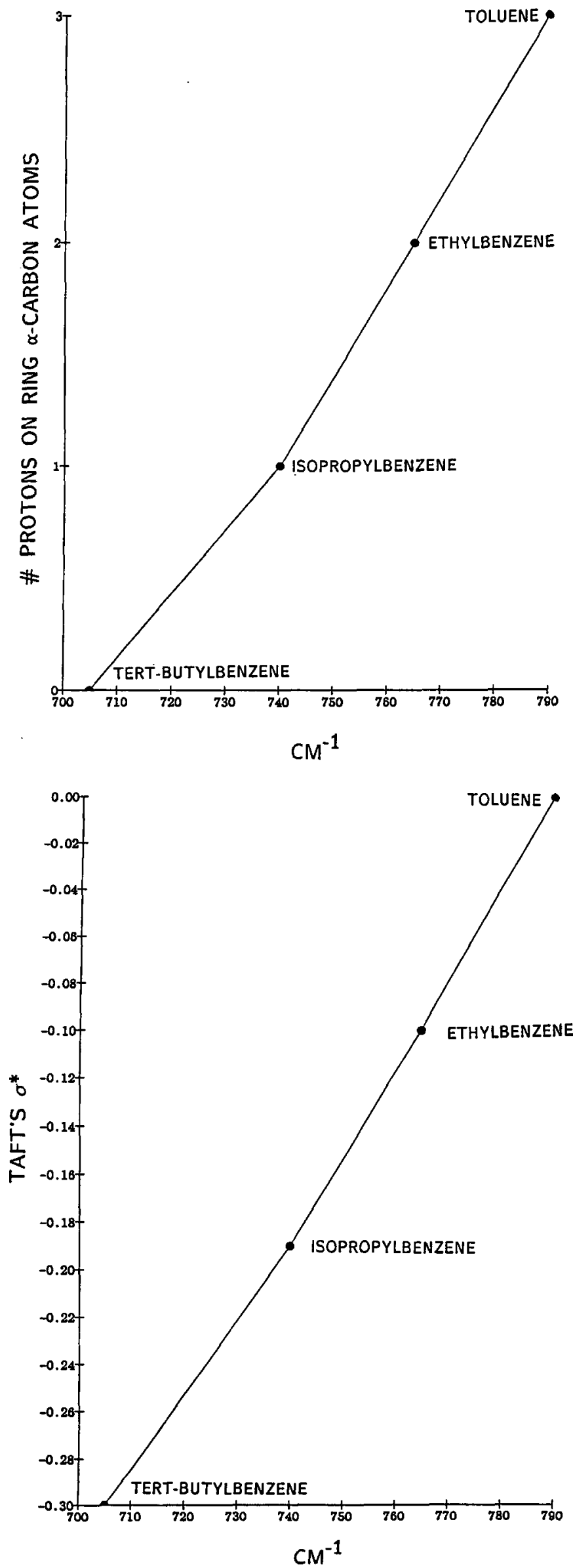

the methyl-to-ring bond. The 790- $\mathrm{cm}^{-1}$ Raman band for toluene is strong, and it is also polarized. Strong Raman bands which are also polarized are observed near 760 $\mathrm{cm}^{-1}, 740 \mathrm{~cm}^{-1}$, and $705 \mathrm{~cm}^{-1}$ for ethylbenzene, isopropylbenzene, and tert-butylbenzene, respectively. ${ }^{6}$ These bands are assigned to the $A_{1}$ species and presumably result from a fundamental comparable to the $790-\mathrm{cm}^{-1}$ $\mathrm{A}_{1}$ fundamental for toluene. Approximately one third of the potential energy for the toluene $790-\mathrm{cm}^{-1}$ fundamental results from $\mathrm{C}-\mathrm{C}$ stretch of the phenyl-C bond. Thus, one might expect that this $A_{1}$ fundamental would be sensitive to alkyl substitution. Figure 1 is a plot of the number of protons on the ring $\alpha$-carbon atom vs. the bands at $790,760,740$, and $705 \mathrm{~cm}^{-1}$ for toluene, ethylbenzene, isopropylbenzene, and tert-butylbenzene, respectively. The plot shows that the $A_{1}$ mode decreases in frequency with increased branching on the ring $\alpha$ carbon atom. Figure 2 is a plot of these same $A_{1}$ mode frequencies vs. Taft's $\sigma^{*}$ values of $0,-0.10,-0.19$, and -0.30 for methyl, ethyl, isopropyl, and tert-butyl groups, respectively. ${ }^{7}$ The plot shows that the $A_{1}$ fundamental decreases in frequency with increasing electron release of the alkyl group. Thus, as the phenyl-C bond becomes weaker, the $A_{1}$ fundamental shifts to lower frequency.

1. R. A. Nyquist, Spectrochim. Acta 19, 713 (1963).

2. R. A. Nyquist, Correlations Between IR and NMR Data: Carbonyl Frequencies vs. Carboxyl Carbon-13 Chemical Shifts (Sadtler Research Laboratories, Philadelphia, Pennsylvania, 1986).

3. R. A. Nyquist, Appl. Spectrosc. 42, 624 (1988).

4. R. A. Nyquist, Appl. Spectrosc. 42, 854 (1988)

5. C. L. Lau and R. G. Snyder, Spectrochim. Acta 27A, 2073, (1971).

6. Raman Spectra (Sadtler Research Laboratories, Philadelphia, Pennsylvania, 19xx).

7. R. W. Taft, Jr., Steric Effects in Organic Chemistry, M. S. Newman, Ed. (J. Wiley \& Sons, New York, 1956), p. 592.

\section{Estimation of the Effective Dielectric Constant of Cyclodextrin Cavities Based on the Fluorescence Properties of Pyrene-3-Carboxaldehyde}

\author{
K. W. STREET, JR., ${ }^{*}$ and \\ W. E. ACREE, JR. \\ Department of Chemistry, Kent State University, \\ Kent, Ohio 44242
}

Index Headings: Cyclodextrin; Fluorescence of pyrene-3-carboxaldehyde; Dielectric constant.

\section{INTRODUCTION}

The microscopic dielectric constant of the cyclodextrin (CD) interior is a fundamental property of these microheterogeneous environments. Polarity and dielectric

Received 18 April 1988.

* Author to whom correspondence should be sent. 


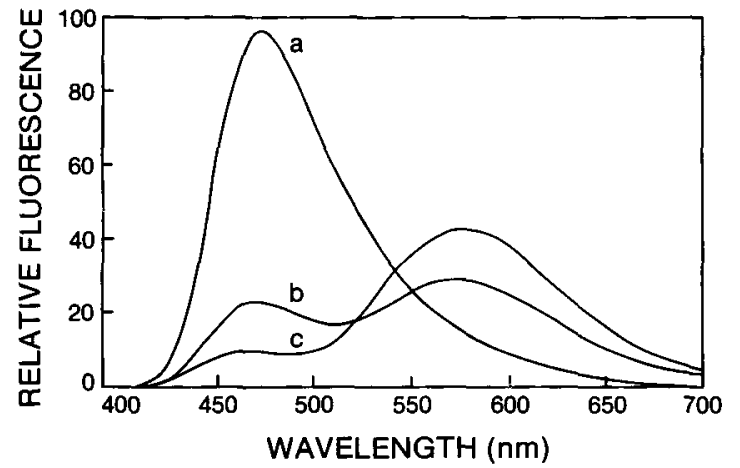

FIG. 1. Fluorescence emission spectra for $\mathrm{PyCHO}$ in (a) water, (b) 1 $\mathrm{mM} \gamma \mathrm{CD}$, and (c) $10 \mathrm{mM} \gamma \mathrm{CD}$.

constant play a crucial role in the understanding of spectroscopy and chromatography. Bulk measurements of these properties are not suitable for the evaluation of inclusion cavities, and frequently luminescent probes are employed to estimate these parameters. Recently, polycyclic aromatic hydrocarbon probes have been used to evaluate the effective polarity ${ }^{1}$ of the CD cavity, and the approximate formation constants ${ }^{2-5}$ were estimated for inclusion complexes of the variety:

$$
\begin{gathered}
\mathrm{PAH}+\mathrm{CD} \rightleftharpoons[\mathrm{PAH}-\mathrm{CD}] \\
{[\mathrm{PAH}-\mathrm{CD}]+\mathrm{CD} \rightleftharpoons[\mathrm{CD}-\mathrm{PAH}-\mathrm{CD}]} \\
{[\mathrm{PAH}-\mathrm{CD}]+\mathrm{PAH} \rightleftharpoons[\mathrm{PAH}-\mathrm{PAH}-\mathrm{CD}]} \\
{[\mathrm{PAH}-\mathrm{CD}]+[\mathrm{PAH}-\mathrm{CD}]} \\
\rightleftharpoons[\mathrm{CD}-\mathrm{PAH}-\mathrm{PAH}-\mathrm{CD}]
\end{gathered}
$$

The common cyclodextrins $(\alpha \mathrm{CD}, \beta \mathrm{CD}$, and $\gamma \mathrm{CD})$ are cyclic oligosaccharides consisting of six $(\alpha)$, seven $(\beta)$ or eight $(\gamma)$ glucose monomers arranged in a torous when dissolved in water. The depth of the CD cavity is approximately $8 \AA$, and the cavity diameter varies with the number of glucose monomers. For $\alpha, \beta$, and $\gamma \mathrm{CD}$ the respective diameters are $4.9,6.2$, and $7.9 \AA .^{6}$ In addition, each cyclodextrin contains numerous primary and secondary hydroxyl groups. The cavity diameter is the primary factor governing the ability of organic molecules to form inclusion complexes. Pyrene is too large to be included in the $\alpha \mathrm{CD}$ cavity, and therefore there is no spectroscopic evidence for inclusion complexing with this system. For $\beta$ CD the cavity is large enough to allow complexes described in Eqs. 1 and 2, and $\gamma \mathrm{CD}$ is large enough to include the pyrene dimer, which is a predominate species at high CD concentrations. ${ }^{1}$

By close inspection of the vibronic fine structure in the fluorescence emission spectrum of pyrene, Kusumoto was able to evaluate the formation constants for Eqs. 1 and 2 between pyrene and $\beta C D .^{2}$ Kobayashi et al. combined similar luminescence data with spectropolarimetric data and obtained an estimate of the formation constants for the four pyrene- $\gamma \mathrm{CD}$ inclusion complexes described in Eqs. 1-4. ${ }^{3}$

Pyrene (Py) and other PAHs were used as polarity probes for solvents, ${ }^{7-9}$ organized media (e.g., micro-emulsions, ${ }^{10,11}$ micelles, ${ }^{12-18}$ and $\mathrm{CDs}^{1-5}$ ) and diverse micro-environment studies. ${ }^{19,20}$ The Py scale (as well as other PAH scales) is based on a change in fluorescence intensity

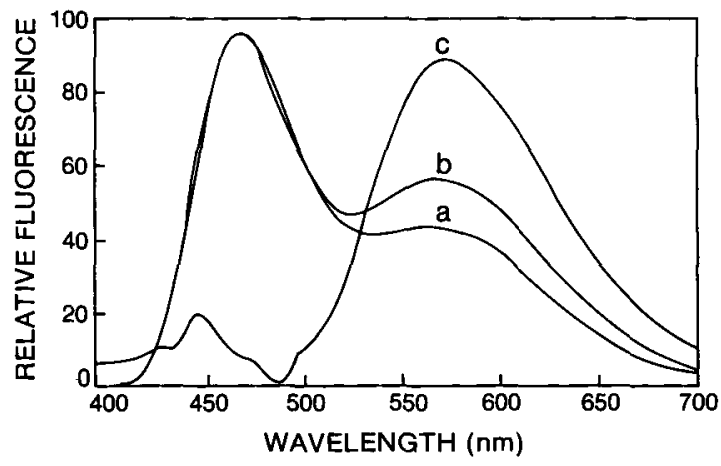

FIg. 2. Fluorescence emission spectra of PyCHO in $0.5 \mathrm{mM} \gamma \mathrm{CD}$. (a) Freshly prepared sample. (b) Sample aged $24 \mathrm{~h}$. (c) Difference between $a$ and $b$.

ratio of the $0-0$ to $0-2$ vibronic bands in the emission spectrum, which are ultimately related to the microscopic polarity about the PAH probe molecule. In this paper the fluorescence probe pyrene-3-carboxaldehyde is used to spectroscopically examine the CD systems. The PyCHO system is extensively characterized and for $\mathrm{ROH}$ (other than acids) containing solvents, the dielectric constant, $\epsilon$, of the medium is highly correlated to the wavelength of maximum fluorescence emission intensity. ${ }^{13,14,17}$ The emission spectrum of PyCHO is composed of two closely spaced bands $\left(\pi^{*}-\pi\right.$ and $\left.\pi^{*}-n\right)$ which vary in intensity. The resulting wavelength of maximum emission therefore varies with their position and intensity, which is in turn correlated to $\epsilon_{.}^{21}$ The PyCHO has inclusion behavior similar to that of Py in CD systems, as it has the same approximate molecular dimensions with respect to CD cavity dimensions.

\section{EXPERIMENTAL}

Materials and Sample Preparation. The $\alpha, \beta$, and $\gamma \mathrm{CD}$ obtained from Advanced Separation Technologies Inc. were used as received. Pyrene-3-carboxaldehyde, obtained from Aldrich Chemical Company, was recrystallized three times from absolute ethanol prior to use. A $2.9 \times 10^{-4} \mathrm{M}$ stock PyCHO solution in cyclohexane was prepared, and small aliquots were evaporated to dryness prior to addition of aqueous CD. All PyCHO concentrations in CD-containing solvents were kept at less than $10^{-5} \mathrm{M}$ to minimize inner filtering artifacts. All solutions were prepared in HPLC quality water (Fisher Scientific).

Measurements. Fluorescence emission spectra were recorded on a Perkin-Elmer LS-5 spectrofluorometer with 3600 data station. All samples were excited at $380 \mathrm{~nm}$ in 1-cm ${ }^{2}$ quartz cuvettes, and the emission spectrum was scanned over at least $\pm 20 \mathrm{~nm}$ of the wavelength of the emission maximum. All wavelengths reported represent the average of at least 5 scans which were blank corrected. Additional measurements were made for $\mathrm{PyCHO}$ dissolved in water, methanol, octanol, and binary aqueous/ methanol mixtures for construction of a calibration plot of $\epsilon$ vs. $\lambda_{\max }$.

\section{RESULTS AND DISCUSSION}

Figure 1 illustrates the uncorrected spectra of $\mathrm{PyCHO}$ in aqueous $\gamma \mathrm{CD}$ and in water. It is apparent that as the 


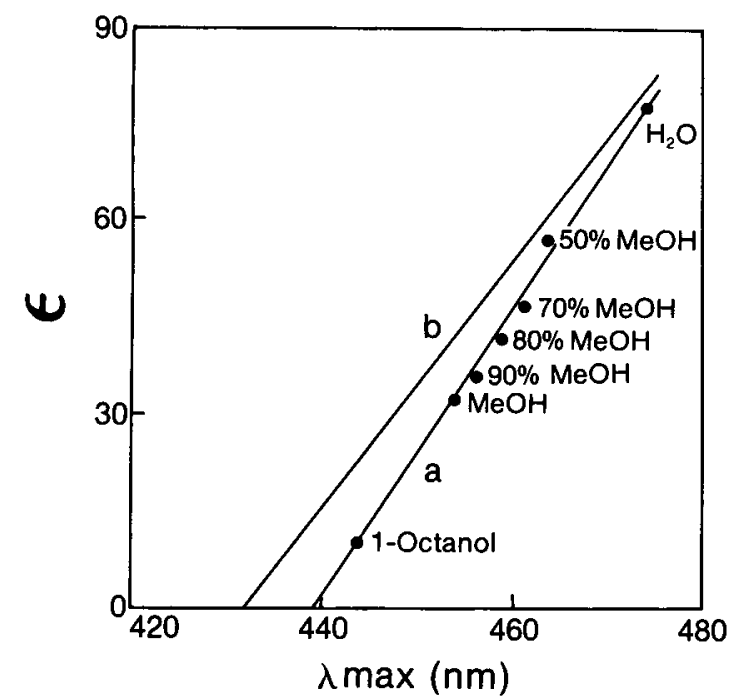

FIG. 3. Plot of wavelength of maximum fluorescence emission intensity vs. dielectric constant. (a) Experimental curve for this work. (b) Published curve generated from Eq. 5 .

$\gamma \mathrm{CD}$ concentration increases, the concentration of PyCHO dimer (peak at $575 \mathrm{~nm}$ ) also increases. According to the Py literature, the fully end-capped PAH (represented by Eq. 4) is the preferred configuration under these conditions. ${ }^{3}$ The formation of the most stable complex is shown in Fig. 2, where spectra $a$ and $b$ are normalized to the height of the monomer peak appearing in the $470-\mathrm{nm}$ region. Figure $2 \mathrm{a}$ was taken at the time of sample preparation, and $2 \mathrm{~b}$ was recorded after $24 \mathrm{~h}$ of aging. Attempts to obtain useful rate constant information associated with the monomer-to-dimer conversion process were hampered by only small differences in the spectra and instrument drift over the course of time required to acquire necessary intensity information for the $470-\mathrm{nm}$ peak. The process is further complicated by equilibrium expressed in Eqs. 1 and 2, which occurs simultaneously with dimer formation and is evidenced by the maxima $(446 \mathrm{~nm})$ and minima $(485 \mathrm{~nm})$ in the amplified difference spectrum, 2c. An expansion of the spectra in the 440-500-nm region indicates that the $470-\mathrm{nm}$ peak is shifted by less than $3 \mathrm{~nm}$ in the process. Figure $2 \mathrm{c}$ further reveals that the dimer spectrum will not significantly distort the monomer spectrum with respect to the wavelength of maximum emission intensity. This observation was independently confirmed by a selective excitation experiment where a clean dimer emission spectrum was obtained and indicates that the intensity of the dimer spectrum is zero at wavelengths of less than $480 \mathrm{~nm}$. The spectra for PyCHO in $\beta \mathrm{CD}$ show no appearance of the dimer peak, suggesting that the cavity is large enough for inclusion of PyCHO but not the dimer. This observation is in line with the literature on Py-CD systems. ${ }^{2}$

The PyCHO dielectric constant measurement is well documented in the literature. For nonacid compounds containing $\mathrm{ROH}$ functionalities:

$$
\lambda_{\max }=0.52 \epsilon+431.5
$$

where $\epsilon$ is the dielectric constant and $\lambda_{\max }$ is the wavelength of maximum emission of the PyCHO monomer.

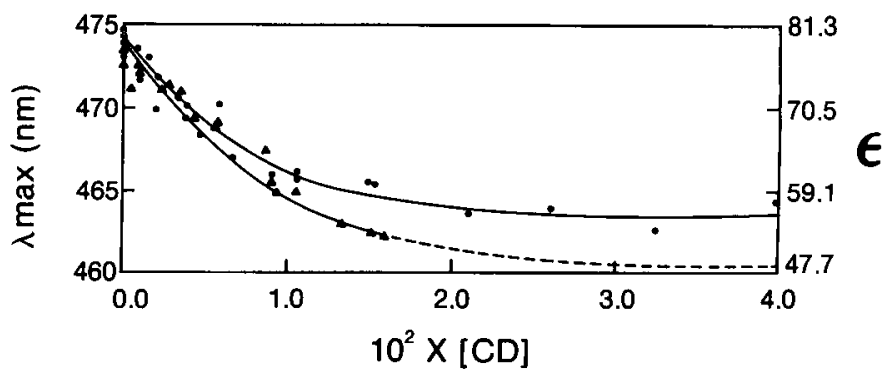

Fig. 4. Plot of CD concentration vs. wavelength of maximum fluorescence emission intensity (left axis) or dielectric constant (right axis). $(\bullet) \gamma \mathrm{CD}$ and $(\triangle) \beta \mathrm{CD}$.

Figure $3 \mathrm{a}$ is a calibration plot for data obtained on our instrument, and $3 \mathrm{~b}$ is a reference plot of Eq. 5. ${ }^{13,14,17}$ The difference between the two plots may be due to differences in blank corrections, spectral slit widths employed, inner filtering (the solutions that we employed were considerably $<10^{-5} \mathbf{M}$, as reported by others), or other artifacts between laboratories. The equation which best describes Fig. 3a is:

$$
\lambda_{\max }=0.44 \epsilon+439 .
$$

Plots of $\lambda_{\max }$ vs. [CD] approach a constant value as the $\mathrm{CD}$ concentration increases (see Fig. 4). In this portion of the plot either the PyCHO or its dimer is fully endcapped, and a reliable estimate of the dielectric constant is possible. Cyclodextrins have a large number of primary and secondary alcohol groups; therefore, the dielectric constant of the cavity is directly calculable from Eq. 6 . The estimated dielectric constants of 55 for $\gamma \mathrm{CD}$ and 48 for $\beta \mathrm{CD}$ are only slightly different. The larger $\gamma \mathrm{CD}$ cavity may possibly include more water molecules, thus producing a slightly larger effective dielectric constant. This conclusion seems reasonable, as the cavity interior of both cyclodextrins contains similar functional groups but slightly differing cavity sizes.

\section{ACKNOWLEDGMENTS}

The authors wish to acknowledge partial support for this research through a Department of Defense University Research Instrument Program of the Army Research Office under Grant DAAL03-87-G-0045. Acknowledgment is also made to the National Institutes of Health Biotechnology Resources Program (Grant 1-R03-RR03145-01) and to the Donors of the Petroleum Research Fund, administered by the American Chemical Society, for partial support of this research. The authors thank Arla White for technical assistance in preparing the manuscript for publication.

1. K. W. Street, Jr., J. Liq. Chromatogr. 10, 655 (1987).

2. Y. Kusumoto, Phys. Chem. Lett. 136, 535 (1987).

3. N. Kobayashi, R. Saito, H. Hino, Y. Hino, A. Ueno, and T. Osa, J. Chem. Soc. Perkin Trans. II, 1031 (1983).

4. A. Nakajima, Spectrochim. Acta, Part A, 39, 913 (1983)

5. A. Nakajima, Bull. Chem. Soc. Japan, 57, 1143 (1984).

6. J. Szejtli, in Inclusion Compounds, J. L. Atwood, J. E. D. Davies, and D. D. MacNicol, Eds. (Academic Press, New York, 1984), Vol. 3, Chap. 11.

7. D. C. Dong and M. A. Winnik, Photochem. Photobiol. 35, 17 (1982).

8. D. C. Dong and M. A. Winnik, Can. J. Chem. 62, 2560 (1984).

9. K. W. Street, Jr., and W. E. Acree, Jr., J. Liq. Chromatogr. 9, 2799 (1986).

10. R. Zana, S. Yiv, C. Strazielle, and P. Lianos, J. Colloid Interface Sci. 80, 208 (1981). 
11. R. B. Thompson and S. E. Shaw McBee, Langmuir 4, 106 (1988).

12. K. Kalyanasundaram and J. K. Thomas, J. Am. Chem. Soc. 99, 2039 (1977).

13. K. Kalyanasundaram and J. K. Thomas, J. Phys. Chem. 81, 2176 (1977).

14. N. J. Turro and T. Okubo, J. Phys. Chem. 86, 159 (1982).

15. N. J. Turro and T. Okubo, J. Am. Chem. Soc. 103, 7224 (1981).

16. P. Levitz, H. Van Damme, and D. Keravis, J. Phys. Chem. 88, 2228 (1984).
17. K. P. Ananthapadmanabhan, E. D. Goddard, N. J. Turro, and P. L. Kuo, Langmuir 1, 352 (1985).

18. N. J. Turro and P. L. Kuo, J. Phys. Chem. 90, 837 (1986).

19. J. Stahlberg and M. Almgren, Anal. Chem. 57, 817 (1985).

20. J. W. Carr and J. M. Harris, Anal. Chem. 58, 626 (1986).

21. K. Bredereck, Th. Forster, and H. G. Oenstein, in Luminescence of Organic and Inorganic Materials, H. P. Kallman and G. M. Spruch, Eds. (Wiley, New York, 1960). 